\title{
Research of TDOA NLOS error compensation interpolation positioning
}

\author{
Yi Yang ${ }^{a}$, Zhongliang Deng ${ }^{b}$
}

School of Beijing University of post and telecommunication, Beijing 100876, China

a774652265@qq.com, bdengzhl@bupt.edu.cn

Keywords: wireless positioning, NLOS compensation, feature point, interpolation.

\begin{abstract}
In the base station positioning system, complex environment will cause reflection and refraction, etc. When using ordinary TDOA positioning algorithm will lead to an inaccurate positioning result in NLOS (Non Line of Sight) environment. In order to improve the positioning accuracy, this paper introduces a TDOA NLOS error compensation interpolation positioning algorithm. In this algorithm, a TDOA NLOS error compensation model is established by using feature point to do interpolation, and use this model together with Newton iterative method to calculate the position we want. This algorithm is tested can reach meter-level positioning accuracy.
\end{abstract}

\section{Introduction}

With the development of information society, the demand for accurate positioning information has become widespread and urgent. Such as shopping malls, parking lots, fire, mine, and other environments, accurate positioning information plays a significant role in our daily life.

This paper introduces a TDOA NLOS error compensation positioning algorithm based on base-station wireless positioning system. In this algorithm we use some feature points to establish the TDOA NLOS error compensation model. List the equations with this model and calculate the positioning result using Newton iterative method. It is proved that by using this algorithm we can get higher accuracy positioning result.

\section{TOA and TDOA positioning}

\subsection{TOA positioning}

TOA (time of arrival), is widely used in wireless positioning. By measuring the spread time from terminal to the base-station, we can list equations of circle with the TOA we measured on terminal (The number of equations is the number of TOAs the terminal get). We can calculate the position of the terminal by solving the equations.

As figure. 1 shows, $d_{n}$ is the TOA of base-station $n$, the equations is as follows:

$$
\left\{\begin{array}{l}
\mathrm{TOA}_{0}=d_{0} \\
\mathrm{TOA}=d_{1} \\
\mathrm{TOA}_{2}=d_{2} \\
\mathrm{TOA}_{3}=d_{3}
\end{array}\right.
$$

In the equations, $d_{i}=\sqrt{\left(x_{i}-x\right)^{2}+\left(y_{i}-y\right)^{2}},\left(x_{i}, y_{i}\right)$ is the coordinate of base-station n, and $(x, y)$ is the coordinate of terminal, the unknown we want to know.

\subsection{TDOA positioning}

TDOA ${ }^{[1]}$, Time difference of arrival, is another positioning method we often use. In TOA positioning method, it is hardly to ensure that the clock of terminal and the clock of base-station are synchronized.

But the problem doesn't exist in TDOA positioning method. Because when we minus TOA $A_{n}$ with $T O A_{m}$ to get the TDOA, the error between terminal clock and base-station clock is eliminated. Based on TDOA principle, we can list equations of hyperbola with the TDOA we measured on terminal 
(The number of equations is the number of TOAs the terminal get). We can get the position of the terminal by solving the equations.

As figure. 1 shows, $d_{n}$ is the TOA of base-station $\mathrm{n}$, the equations is as follows:

$$
\left\{\begin{array}{l}
\mathrm{TDOA}_{10}=\left(d_{1}-d_{0}\right) \\
\mathrm{TDOA}_{20}=\left(d_{2}-d_{0}\right) \\
\mathrm{TDOA}_{30}=\left(d_{3}-d_{0}\right)
\end{array}\right.
$$

In the equation, $\left(x_{n}, y_{n}\right)$ is the coordinate of base-station $\mathrm{n}$, and $(x, y)$ is the coordinate of terminal, the unknown we want to know.

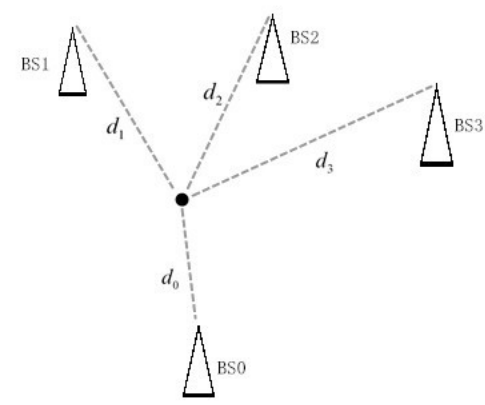

Fig. 1 Wireless positioning principles

\section{TDOA NLOS error compensation interpolation model}

\subsection{Non Line of Sight Propagation}

NLOS: Many types of radio transmissions depend, to varying degrees, on line of sight (LOS) between the transmitter and receiver. Obstacles that commonly cause NLOS conditions include buildings, trees, hills, mountains, and, in some cases, high voltage electric power lines. Some of these obstructions reflect certain radio frequencies, while some simply absorb or garble the signals; but, in either case, they limit the use of many types of radio transmissions, especially when low on power budget. $^{[2]}$

In city positioning environment, NLOS is very serious, and it cause great error in wireless positioning system.

\subsection{Feature Point}

Feature point is some points that we already know their position coordinate, and we also know some information of the point that is related with wireless positioning. In TDOA positioning, we use our terminal to collect the TDOA in some points that we already know there position coordinate, and store their position coordinate and TDOA information into the feature point information database to help us solving the NLOS problem.

\subsection{TDOA NLOS error compensation}

TDOA NLOS error compensation ${ }^{[3]}$ is the difference between the TDOA of LOS and the TDOA of NLOS in a certain point.

As Figure 2 shows, the distance between terminal and BS0, BS1 (two base-stations) are $d_{0}$ and $d_{1}$. TDOA in LOS, $T D O A_{10}=d_{1}-d_{0}=T O A_{1}-T O A_{0}$, but in real environment, the electromagnetic wave is blocked in LOS, it can only reach the terminal by reflection (the green line), NLOS, so the NLOS TDOA TDOA ${ }_{10}{ }^{\prime}=d_{1}{ }^{\prime}-d_{0}{ }^{\prime}, d_{n}{ }^{\prime}$ is the NLOS distance between base-station $\mathrm{n}$ and terminal. The TDOA NLOS error compensation:

$$
T C=T D O A_{10}-T_{D O A}{ }_{10}^{\prime}
$$




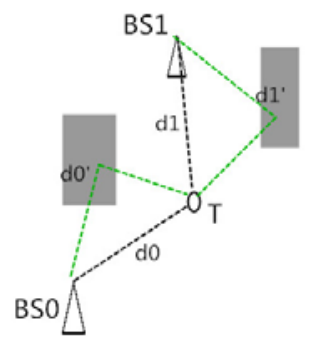

Fig. 2 NLOS principles

\subsection{TDOA NLOS error compensation interpolation models}

Positioning environment can be divided into two types: straight type and area type.

We use the feature points' information to establish a TDOA NLOS error compensation model: we trait the environment position coordinates as independent variables, we trait the TDOA NLOS error compensation as dependent variables.

$T C(x)=G(x)$

Here, $\mathrm{x}$ is position coordinates. $G(x)$ is the model we established, $T C(x)$ is TDOA NLOS error compensation that is related to the position coordinates.

TDOA NLOS compensation in Straight type environment

The relationship of the distance of a point outside a line to a certain point on the line is a hyperbola when we trait the line as $\mathrm{X}$-axis in two-dimension coordinate system. We tested that, quadratic piecewise Hermite interpolation polynomial can be similar to the hyperbola. And TDOA NLOS error compensation is linear combination of TDOA, and TDOA is also a linear combination of TOA. So TDOA NLOS error compensation is linear combination of TOA. So the quadratic piecewise Hermite interpolation polynomial can be similar to a model of TDOA NLOS error compensation.

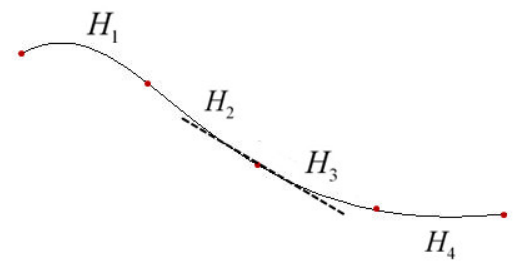

Hermite interpolation ${ }^{[4]}$ :

Fig. 3 two-times piecewise Hermite interpolation

In order to make the interpolation polynomial more similar to the original function, we not only make them the same value at the node but also make their derivative the same value. We call this kind of interpolation Hermite interpolation, and the interpolation polynomial Hermite polynomial.

$$
\begin{aligned}
& \left\{\begin{array}{l}
H_{i}\left(\mathrm{x}_{i}\right)=\text { Tcom }_{i} \quad i=0,1,2,3 \ldots \\
H_{i}\left(\mathrm{x}_{i+1}\right)=\text { Tcom }_{i+1} \quad i=0,1,2,3 \ldots \\
H_{k}^{\prime}\left(\mathrm{x}_{k}\right)=\text { Tcom }_{k}^{\prime} \quad \mathrm{k}=1,2,3 \ldots, \mathrm{n} \\
H_{k}^{\prime}\left(\mathrm{x}_{k+1}\right)=\text { Tcom }_{k+1}^{\prime} \quad \mathrm{k}=0,2,3 \ldots, \mathrm{n}-1
\end{array}\right. \\
& H(\mathrm{x})=\sum_{j=0}^{n}\left[\mathrm{y}_{j} \mathrm{a}_{j}(\mathrm{x})+\operatorname{Tcom}_{j} \mathrm{~b}_{j}(\mathrm{x})\right] \\
& \mathrm{a}_{j}(\mathrm{x})=\left[1-2\left(\mathrm{x}-\mathrm{x}_{j}\right) \sum_{\substack{k=0 \\
k \neq j}}^{n} \frac{1}{x_{j}-x_{k}}\right] \mathrm{l}_{j}^{2}(\mathrm{x}) \\
& \mathrm{b}_{j}(\mathrm{x})=\left(\mathrm{x}-\mathrm{x}_{j}\right) \mathrm{l}_{j}^{2}(\mathrm{x}) \\
& \mathrm{l}_{j}(\mathrm{x})=\frac{\prod_{\substack{i=0 \\
i \neq j}}^{n}\left(\mathrm{x}-\mathrm{x}_{i}\right)}{\prod_{\substack{i=0 \\
i \neq j}}^{n}\left(\mathrm{x}_{j}-\mathrm{x}_{i}\right)}
\end{aligned}
$$


$H_{i}(\mathrm{x})$ is the Hermite interpolation polynomial. We set of the tangent line of middle feature points the perpendicular of the angel bisector which the angle is the angel between the two line of center feature point to other two feature points near by it to make the polynomial curve smoother.

Use the Lagrange interpolation base function to solve equation (4):

When we have three or more feature points, we can use Hermite interpolation to establish the TDOA NLOS error compensation model.

When we only have one or two feature points:

Own feature point: we see the TDOA NLOS error compensation of this point as TDOA NLOS error compensation in all the straight line positioning environment.

Two feature point: we use the TDOA NLOS error compensation of the two points represent the TDOA NLOS error compensation in the straight line environment linearly.

TDOA NLOS error compensation in Area type environment

We tested that, double B spline interpolation ${ }^{[5]}$ surface can be similar to a TDOA NLOS error compensation in an area positioning environment.

Double B spline interpolation surface

$$
\begin{aligned}
& \bar{r}_{l, k}(u, v)=\sum_{i=0}^{3} \sum_{i=0}^{3} E_{i, 3}(\mathrm{u}) E_{j, 3}(v) r_{(i+l)(j+\mathrm{k})} \\
& 0 \leq u, v \leq 1, L=01, \ldots, 3 n-1, K=0,1, \ldots, 3 m-1
\end{aligned}
$$

The base function is:

$$
\begin{aligned}
& E_{0,3}(t)=\left(-t^{3}+3 t^{2}-2 t+1\right) / 3 !, \\
& E_{1,3}(t)=\left(3 t^{3}-6 t^{2}+4\right) / 3 !, \\
& E_{2,3}(t)=\left(-3 t^{3}+2 t^{2}+3 t+1\right) / 3 !, \\
& E_{3,3}(t)=t^{3} / 3 !
\end{aligned}
$$

\begin{tabular}{|c|c|}
\hline $\begin{array}{c}\text { Number of feature } \\
\text { points }\end{array}$ & TDOA NLOS error Compensation model algorithm \\
\hline 1 & $\begin{array}{l}\text { Trait TDOA NLOS error compensation of this point as TDOA NLOS error } \\
\text { compensation in all area. }\end{array}$ \\
\hline 2 & $\begin{array}{l}\text { Make a plane through these two feature points, the intersect line with the area } \\
\text { plane is vertical to the line through two featrue points. Trait this plane as the } \\
\text { TDOA NLOS error compensation in this area. }\end{array}$ \\
\hline 3 & $\begin{array}{l}\text { Make a plane through these three feature points, Trait this plane as the TDOA } \\
\text { NLOS error compensation in this area. }\end{array}$ \\
\hline $4 \sim 8$ & $\begin{array}{c}\text { Use 2-times polynomial surface interpolation, and make it the TDOA NLOS } \\
\text { error compensation in this area. }\end{array}$ \\
\hline
\end{tabular}

More detail calculation in reference [5].

But if we want to use this interpolation the feature points should be no less than 9 .

When we do not have 9 feature points, we can use other method to establish the model. Show as table 1.

Table 1 TDOA NLOS error compensation model with less than 9 feature points

\section{Positioning calculation and result}

\subsection{Positioning calculation algorithm}

According to the TDOA NLOS error compensation model we established in Chp.3, $T C=G(\mathrm{x})$, the following equations can be listed:

$$
\left\{\begin{array}{l}
\operatorname{TDOA}_{10}-G_{10}(X)=F_{10}(X) \\
\cdots \\
\text { TDOA }_{i 0}-G_{i 0}(X)=F_{i 0}(X)
\end{array}\right.
$$


$T D O A_{i 0}$ is the TDOA we actually measured in the a certain position(NLOS TDOA). $\mathrm{X}$ is the terminal position we want calculate, unknown. $\mathrm{F}(\mathrm{X})$ is LOS TDOA in a certain position $\mathrm{X}$.

$F_{i 0}(x)=\sqrt{\left(x_{i}-x\right)^{2}+\left(y_{i}-y\right)^{2}}-\sqrt{\left(x_{0}-x\right)^{2}+\left(y_{0}-y\right)^{2}}$

$\left(x_{n}, y_{n}\right)$ is the coordinate of base-station $\mathrm{n}$, and $(x, y)$ is the coordinate of terminal, the unknown we want to know.

We can solve the equation (8) by using the Newton iterative method. When using the Newton iterative method ${ }^{[6]}$ we need an original point to begin the iterative, here we can choose a feature point as the original iterative point.

\subsection{Result}

By using the algorithm we introduced in 4.1, the result we actually test and calculated is as follow:

RMSE of TDOA is 10ns, using the algorithm the paper introduce to get the result, and compare the result with the actual coordinate, and getting the CDF of positioning error as follow:

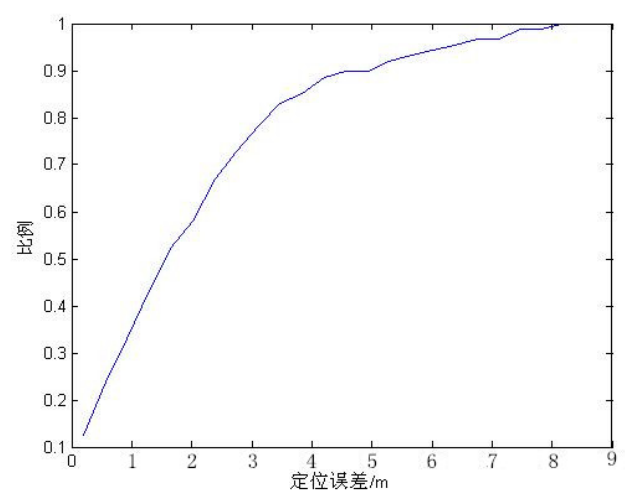

Fig. 3 CDF of positioning error

The $\mathrm{X}$-axis is positioning error in meter, $\mathrm{Y}$-axis is a proportion: $\mathrm{C} / \mathrm{A}$

$\mathrm{C}$ is the number of point which the positioning error is no more than the corresponding error in $\mathrm{x}$-axis. A is the number of all points we tested.

\section{Summary}

This paper introduced an algorithm, in this algorithm we use TDOA NLOS error Compensation model to fix the NLOS error in complex positioning environment. By using this algorithm, we can get high accuracy positioning result when the wireless signal is relatively stable. In general this algorithm is suitable for city positioning.

\section{Acknowledgement:}

This work is supported by National High Technology Research and Development Program of China (NO.2014AA123103, NO.2015AA124103)

\section{References}

[1]. Xiaohong Shi. TDOA-based wireless positioning method and its performance [J]. Journal of Southeast University (Natural Science Edition). Vol. 43 (2013) No. 2, p. 252-257

[2]. Information on: https://en.wikipedia.org/wiki/Non-line-of-sight_propagation

[3]. Thesis: (Xiaohong Zhao: Research on mobile network based NLOS error compensation and key position calculating technology [D] (M.E.E, Beijing University of post and teleommunication, China 2014).

[4]. Xiao-e Wang, Qifang Su, et al. Some type of Hermite interpolation and calculation [J]. Journal of Taizhou University, Vol. 36 (2014) No. 6, p. 1-4 
[5]. Kui Fang. Double 3-times B-spline interpolation suface[J]. Mathematical Theory and Application. Vol. 21 (2001) No. 3, p. 66-68

[6]. Hui Liu. Newton iterative method for non-linear solutions and its application [J]. Journal of Chongqing Institute of Technology (Natural Science Edition). Vol.21 (2007) No. 8, p. 95-98 